\title{
Nuevos registros de ictiosaurios (Reptilia: Ichthyosauria) del Jurásico Superior en Nuevo León, México
}

\author{
New occurrences of ichthyosaurs (Reptilia: Ichthyosauria) of the Upper Jurassic in Nuevo León, \\ Mexico
}

Rogelio Antonio Reyna-Hernández ${ }^{1}$, Luis Encarnación Silva-Martínez ${ }^{1, *}$, Héctor E. Rivera-Sylva ${ }^{2}$

${ }^{1}$ Laboratorio de Paleobiología, Facultad de Ciencias Biológicas, Universidad Autónoma de Nuevo León, Avenida Pedro de Alba y Manuel L. Barragán s/n, Ciudad Universitaria, 66455, San Nicolás de los Garza, Nuevo León, México.

${ }^{2}$ Departamento de Paleontología, Museo del Desierto, Carlos Abedrop Dávila 3745, Saltillo, 25022, Coahuila, México.

* Autor para correspondencia:

(L.E. Silva-Martínez)luis.silva.paleo@gmail.com

\section{Cómo citar este artículo:}

Reyna-Hernández, R.A., Silva-Martínez, L.E., Rivera-Sylva,H.E., 2021, Nuevos registros de ictiosaurios (Reptilia: Ichthyosauria) del Jurásico Superior en Nuevo León, Méxio: Boletín de la Sociedad Geológica Mexicana, 73 (1), A150220. http://dx.doi.org/10.18268/ BSGM2021v73nla150220

Manuscrito recibido: 12 de Noviembre de 2019

Manuscrito corregido: 8 de Enero de 2020

Manuscrito aceptado: 13 de Enero de 2020

La revisión por pares es responsabilidad de la

Universidad Nacional Autónoma de México.

Este es un artículo Open Access bajo la licencia CC $Y-N C$-SA (https://creativecommons.org/licenses/by-nc-sa/4.0/)

\section{RESUMEN}

La diversidad paleontológica de reptiles marinos mesozoicos en el noreste de México demuestra un registro fósil considerable, compuesto por distintos órdenes, incluyendo Ichthyosauria, grupo que presenta reportes en formaciones del Jurásico de la región. Este trabajo presenta dos ejemplares colectados en Galeana, Nuevo León; los cuales están constituidos por elementos postcraneales, procedentes de la Formación La Caja, del Jurásico Superior. Estos complementan el registro fósil de la zona y contribuyen al mayor entendimiento de la diversidad faunística y paleoecológica del Paleo-Golfo de México durante el Jurásico Superior.

Palabras clave: Ichthyosauria, Jurásico Superior, Titoniano, Formación La Caja.

\section{ABSTRACT}

The paleontological diversity of Mesozoic marine reptiles in northeastern Mexico showes an extensive fossil record, composed of different orders, including Ichthyosauria, a group with reports in Jurassic formations of the region. This paper presents two specimens collected in Galeana, Nuevo León, comprised by postcranial elements, from the La Caja Formation, of the Upper Jurassic. The mentioned specimens increase the fossil record of the area and contribute to the greater understanding of the fauna and paleoecological diversity of the PaleoGulf of Mexico during the Upper Jurassic.

Keyzords: Ichthyosauria, Upper Jurassic, Tithonian, La Caja Formation. 


\section{Introducción}

Aunque los restos de reptiles marinos aparecen en varias formaciones en el noreste de México, son generalmente raros y pobremente preservados. Probablemente por este motivo han permanecido como un componente poco estudiado en la asociación faunística.

Diferentes linajes de reptiles colonizaron el ambiente marino durante el Mesozoico. Entre estos, los ictiosaurios fueron el grupo que adquirió el nivel más alto de adaptación al medio acuático. Sus cambios anatómicos incluían cuerpos hidrodinámicos, con una cola en forma de luna y una aleta dorsal. Al formar aletas y la disposición de las extremidades se encontraban fuertemente modificadas, perdiendo el dígito I (Motani, 1999), además se presentaba la disminución del tamaño de los huesos de las extremidades, con excepción del húmero y el fémur, correlacionado con la perdida de osificación pericondral (Caldwell, 1997). La biocronología de los ictiosaurios se extiende desde el Triásico Temprano (Spatiano) hasta el Cretácico Tardío (Cenomaniano) (Motani, 1999; McGowan y Motani, 2003).

Los registros de ictiopterigios en la región son relativamente escasos y se encuentran en los estratos jurásicos de las Formaciones La Caja y La Casita, principalmente en su límite con el Cretácico, donde, por lo menos, se han encontrado dos géneros contemporáneos de la familia Ophthalmosauridae: Brachypterygius y Ophthalmosaurus, y un ejemplar indeterminado perteneciente a esta familia, todos procedentes del estado de Coahuila (Buchy y López-Oliva, 2009; Buchy, 2010; Buchy y Covarrubias, 2011). Asimismo, existe una considerable cantidad de ejemplares fragmentarios y pobremente preservados procedentes de las mismas formaciones geológicas y provenientes de localidades dentro del estado de Nuevo León, últimas que, debido a su falta de elementos diagnósticos, han sido asignadas al clado Thunnosauria (Buchy et al., 2006; Buchy, 2007; Frey y Stinnesbeck, 2014). Por otro lado, fuera del noreste de México, se reporta un ejemplar procedente de la Formación La Casita en Cuencamé, Durango (Frey y Stinnesbeck, 2014). Este ejemplar recientemente fue asignado a su propio género y especie, Acuetzpalin carranzai, el cual también pertenece a la familia Ophthalmosauridae (Barrientos-Lara y AlvaradoOrtega, 2020).

El presente trabajo busca describir e identificar taxonómicamente dos ejemplares fósiles colectados en la región noreste de México, los cuales se encuentran depositados en la colección Paleobiológica del Laboratorio de Paleobiología, de la Facultad de Ciencias Biológicas y proceden de la Formación La Caja, en el Ejido Pablillo, Galeana, Nuevo León.

Abreviaturas institucionales: FCBUANL, Facultad de Ciencias Biológicas, Universidad Autónoma de Nuevo León.

\section{Material y métodos}

Los ejemplares a estudiar proceden de dos afloramientos de la Formación La Caja, localizada en el Ejido Pablillo, Galeana, Nuevo León (Figura 1). La secuencia del área de estudio presenta un miembro con una serie de lutitas calcáreas y margas arcillosas con matices café grisáceos, en la cuales se presentan concreciones calcáreas fosilíferas, similares a la descripción del Miembro D reportado por Rogers et al. (1957) en la localidad tipo de la Formación La Caja; mismo que se localiza debajo del contacto con la Formación Taraises. Sin embargo, la secuencia en el área de estudio aún no ha sido propiamente estudiada. La fauna fósil asociada en la localidad Ejido Pablillo presenta principalmente al amonite Salinites grossicostatum (Figura 2A), el cual permite la determinación de la edad del sitio al Titoniano Tardío, hace 146 millones de años (Imlay, 1939; Adatte, 1994, Zell y Stinnesbeck, 2016). Del mismo modo, se encuentra el pelecípodo Anopaea sp. (Figura 2B), previamente reportado por Zell et al. (2015) para la Formación La Caja en su contacto superior.

El material descrito para este trabajo se encuentra dentro de la colección del Laboratorio de Paleobiología de la Facultad de Ciencias Biológicas en la Universidad Autónoma de Nuevo León. 

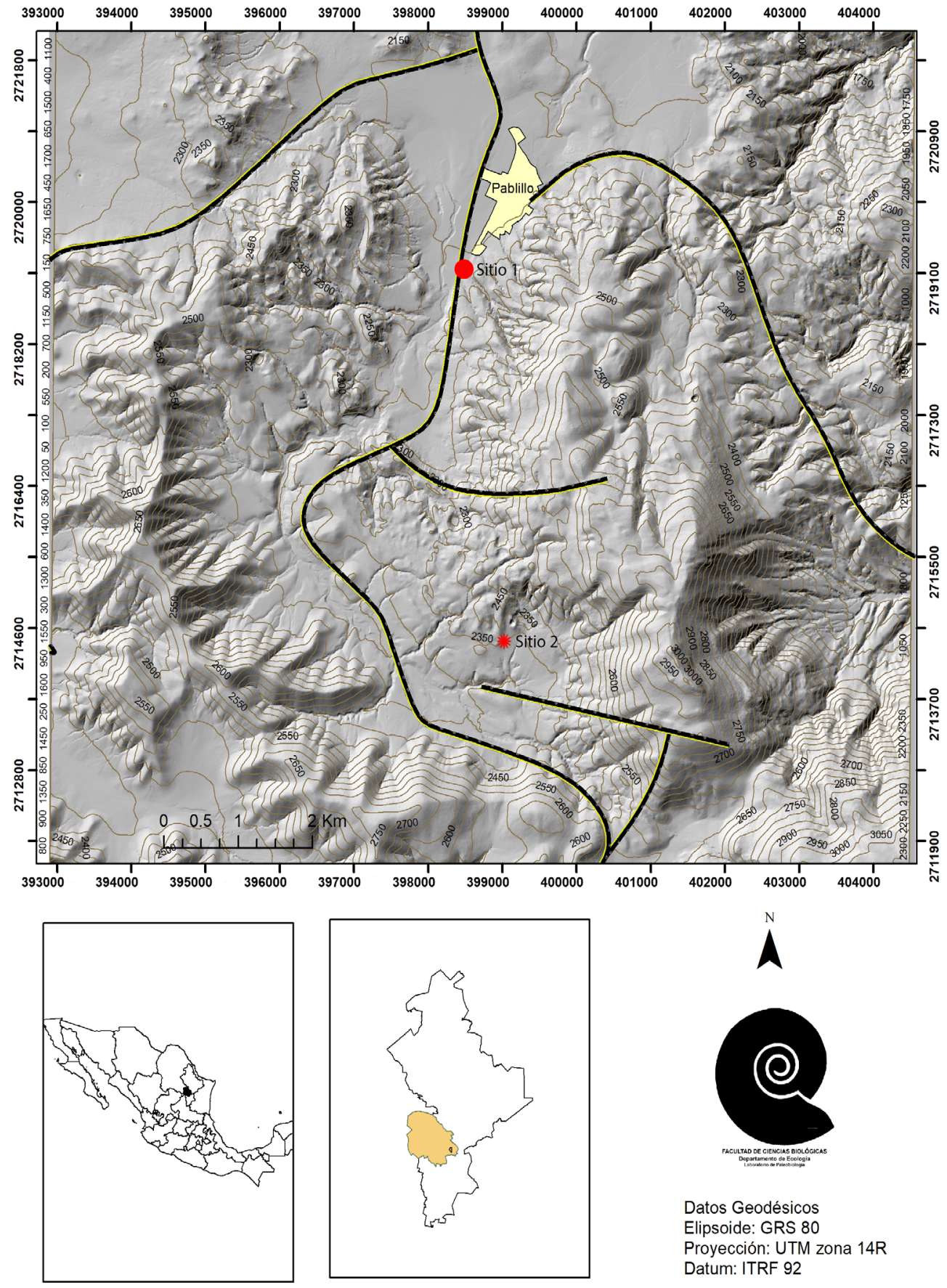

Datos Geodésicos

Elipsoide: GRS 80

Proyección: UTM zona 14R

Datum: ITRF 92 


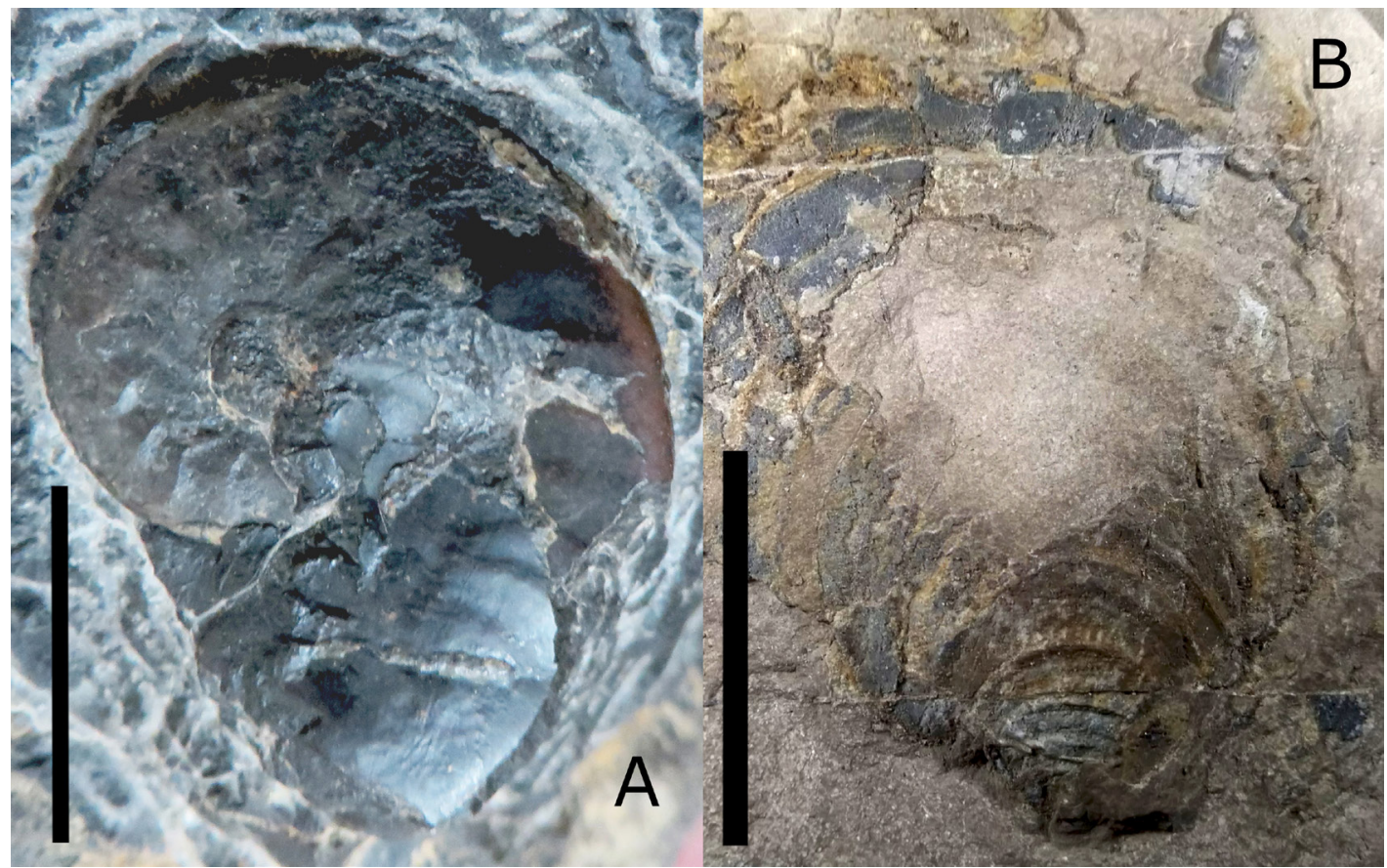

Figura 2 Fauna asociada al espécimen FCBUANL_2709 en el área de estudio: (A) Salinites grossicostatum, escala: 10 milímetros; (B) Anopaea sp., escala: 25 milímetros.

\section{Peleontología sistemática}

Clase Reptilia Laurenti, 1768

Subclase Diapsida Osborn, 1903

Superorden Ichthyopterygia Owen, 1840

Orden Ichthyosauria Blainville, 1835

Clado Thunnosauria Motani, 1999

Gen. et sp. indet.

Material: FCBUANL_2709, dos vértebras parciales articuladas (Figura 3).

Localidad: Ejido Pablillo (Sitio 1), Galeana, Nuevo León, México.

Posición estratigráfica: Formación La Caja, Titoniano Superior (Jurásico Superior).

Descripción: Dos centros articulados de vertebras holóspondilas anficélicas, compactas en sentido craneocaudal y forma discoide. En ambos centros, la parte dorsal presenta una mala pre- servación; debido a esto no se logra apreciar con claridad el canal neural ni la presencia del arco neural. Del mismo modo, presentan un par de apófisis en la parte lateral inferior, en los cuales las costillas (no presentes) se articulan (Figuras 3B y 3C). Se puede apreciar un aspecto cóncavo tanto en la parte ventral como lateral de ambos centros. El centro 1 presenta una longitud de 36 milímetros, una altura máxima de 93 milímetros y un ancho de 101 milímetros; el centro 2, presenta una longitud de 37 milímetros, y una altura máxima de 99 milímetros y un ancho de 99 milímetros.

Familia Ophthalmosauridae Baur, 1877

Gen. et sp. indet.

Material: FCBUANL_2708, 14 vértebras y aleta anterior izquierda parcial (Figura 4).

Localidad: Ejido Pablillo (Sitio 2), Galeana, Nuevo León, México.

Posición estratigráfica: Formación La Caja, Titoniano Superior (Jurásico Superior).

Descripción: 14 centros vertebrales articulados holóspondilos anficélicos, compactos en sentido craneocaudal y forma discoide; los 10 primeros con una erosión 
fuerte en la parte dorsal, los últimos cuatro con los arcos neurales incompletos (Figura 4). Del quinto al décimo centro se presenta una erosión que permite observar la condición ampliamente anficélica de las vértebras (Figura 5).

Las mediciones de estos elementos se ven comprometidas debido a disposición dentro de una matriz calcárea compacta, la cual dificulta la limpieza adecuada del ejemplar por métodos físicos y químicos, sin embargo, la longitud de centros se presenta entre un rango de los 12 a 14 milímetros. Por su parte, algunos fragmentos de costillas se encuentran a los costados de los centros. Aleta anterior izquierda presente de manera parcial, con varios elementos no identificados, con la excepción de la escápula. El eje de la escapula presenta una longitud de 54 milímetros.

La región de la contribución glenoidea se encuentra erosionada y ausente, aunque se conserva el molde de esta en la matriz; el acromion se encuentra mayormente incrustado dentro de la matriz; el margen anterodorsal es recto; el proceso posterior esta reducido; eje escapular presente proximal y distalmente; el molde de la faceta glenoidea permite observar un ángulo menor a $60^{\circ}$ respecto al eje escapular, al formar una condición casi paralela (Figura $6)$.

\section{Discusión}

Las sinapomorfias establecidas por Motani (1999) y restructuradas por Moon (2019) demuestran que los elementos craneales y apendiculares son los que permiten la identificación de ejemplares a taxa inferiores a Ichthyopterygia al llegar incluso a género. Desafortunadamente, el ejemplar FCBUANL_2709 presenta solamente dos elementos vertebrales, lo cual dificulta su identificación a un taxón inferior.

Buchy et al. (2006) describieron previamente seis ejemplares procedentes de distintas localidades de
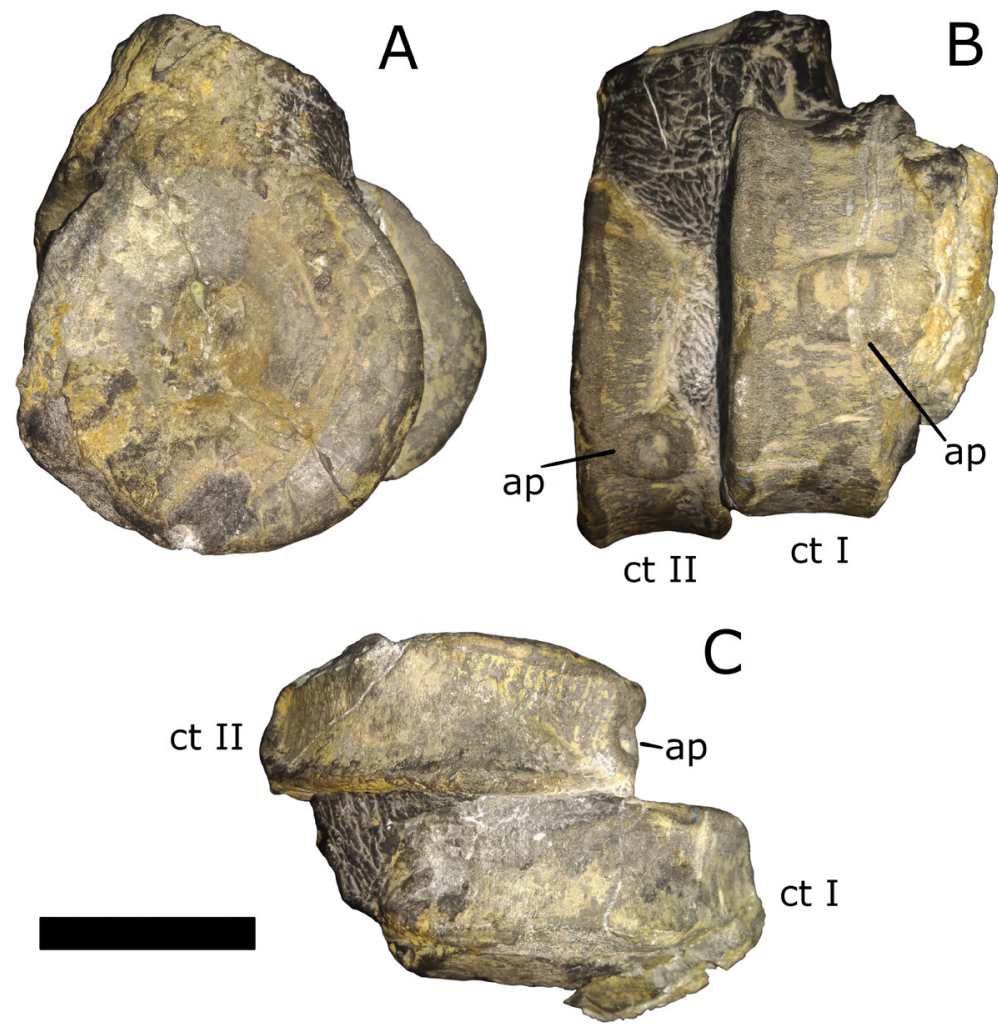

Figura 3 FCBUANL_2709, Thunnosauria indet., fotografías del ejemplar en: (A) vista posterior del centro II; (B) vista lateral derecha; (C) vista ventral. Abreviaciones: ap, apófisis; ct: centro vertebral. Barra de escala: 50 milímetros. 

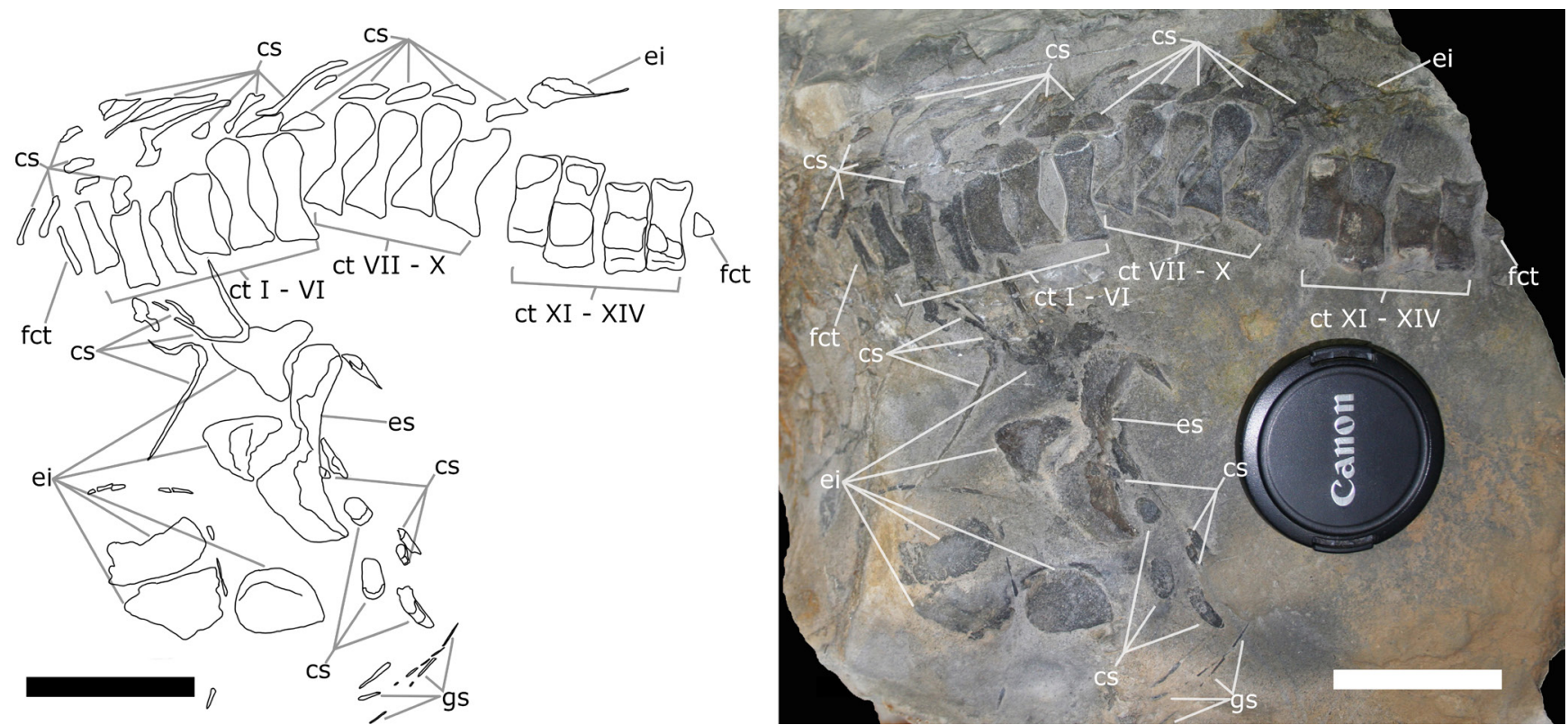

Figura 4 TFCBUANL_2708, Ophthalmosauridae indet., (A) Esquema interpretativo y (B) fotografía del ejemplar. Abreviaciones: ct, centro vertebral; ei, elemento indeterminado; es, escapula; cs, costilla; fct, fragmento de centro vertebral; gs, posible gastralia. Barra de escala: 50 milímetros.

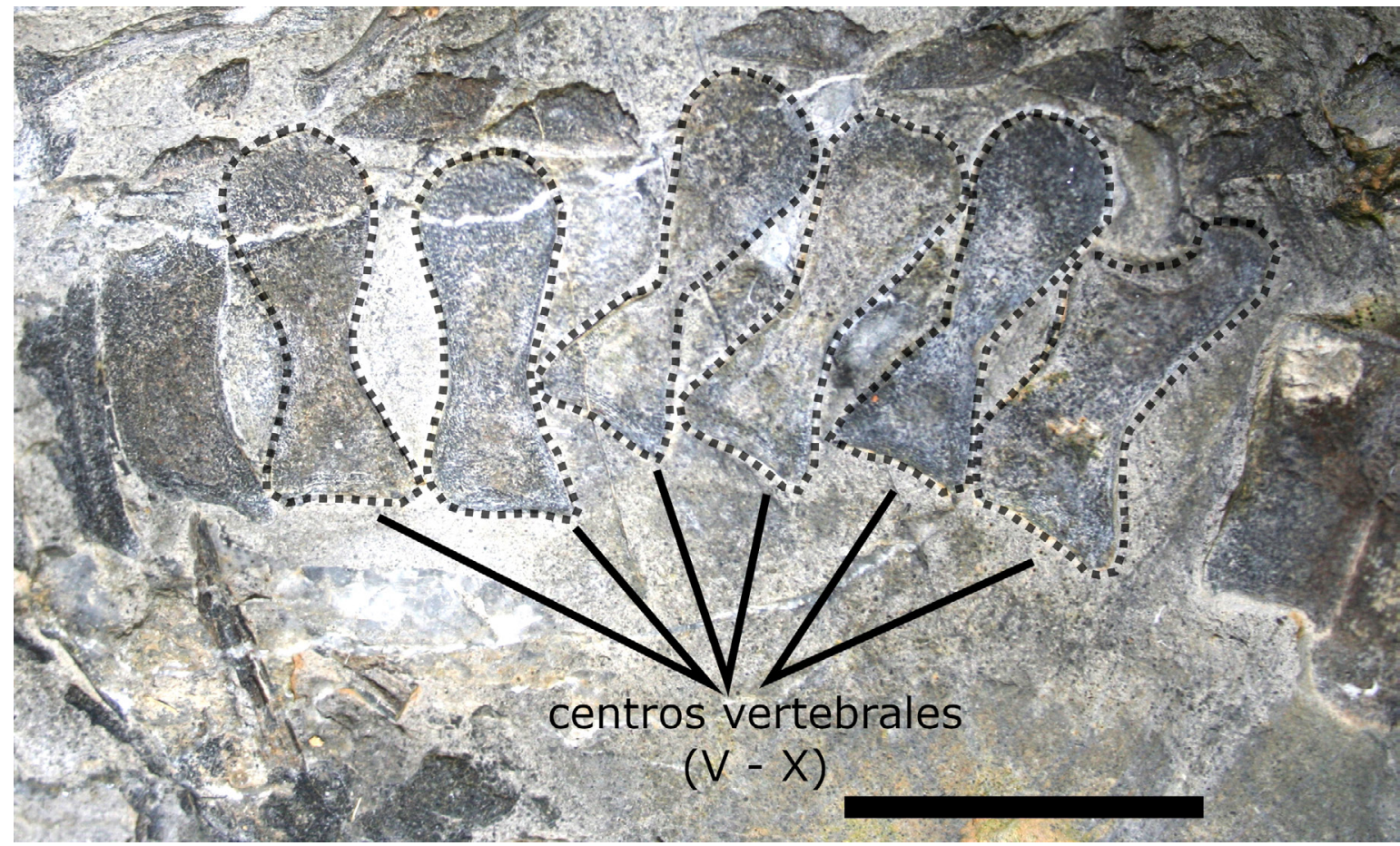

Figura 5 Fotografía de los centros vertebrales V (izquierda) a X (derecha) de FCBUANL_2708. La erosión permite observar la sección longitudinal de los centros, logrando apreciarse la condición anficélica de las vértebras otorgando la forma característica de "reloj de arena". Barra de escala: 25 milímetros. 


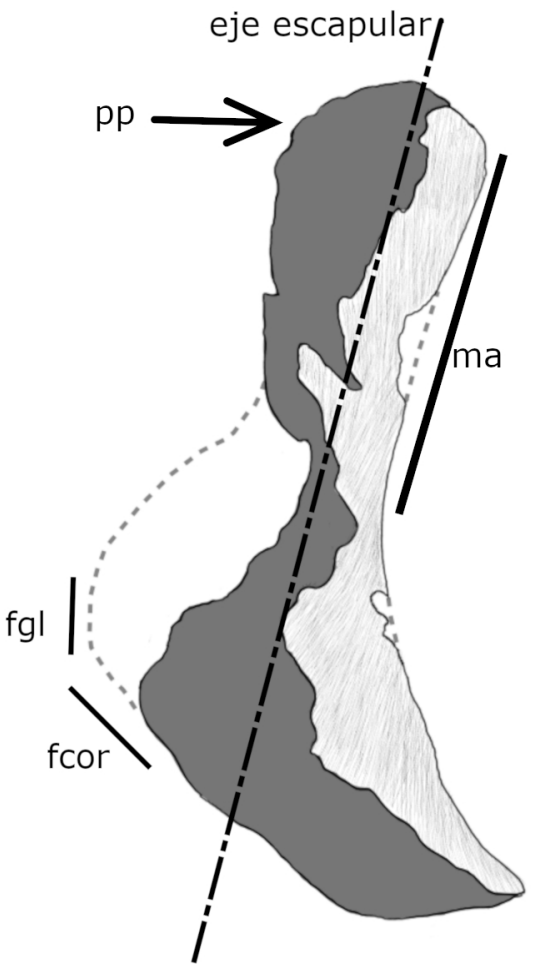

Figura 6 Esquema interpretativo de la escapula de FCBUANL_2708: la zona sombreada indica las partes erosionadas de la escapula; la línea punteada gris indica el molde de la escapula en la matriz; la línea punto-guion negra indica el eje de la escapula. Abreviaciones: fcor, faceta coracoidal; fgl, contribución glenoidea; ma, margen anterodorsal; pp, proceso posterior. Barra de escala 25 milímetros.

las formaciones jurásicas La Casita y La Caja, en la región de la Sierra Madre Oriental al sur del estado de Nuevo León. Debido a la mala preservación de los ejemplares y a la ausencia de elementos craneales y del esqueleto apendicular, no fueron asignadas a un taxón más específico, y fueron relegadas al clado Euichthyopterygia. Posteriormente, Frey y Stinnesbeck (2014) sugirieron que los ejemplares pertenecen al clado más derivado Thunnosauria debido a que es el único grupo de ictiopterigios vivientes durante el Jurásico superior (Motani, 1999). La condición fragmentaria de FCBUANL_2709 asemeja al estatus de los seis ejemplares mencionados anteriormente, debido a la ausencia de material craneal o apendicular asociado.

FCBUANL_2709 presenta únicamente un par de apófisis situadas en la parte inferior de cada costado en cada uno de los centros. McGowan y
Motani (2003) indican que la presencia de un solo par de apófisis situados en la parte inferior lateral en el centro vertebral es propia de las vértebras anterocaudales en el superorden Ichthyopterygia. Asimismo, Maisch y Matzke (2000) indican que en mixosaurios, shastasaurios e ictiosaurios postriásicos, las vértebras caudales son, sin excepciones, más altas que largas.

En cuanto al espécimen FGBUANL_2708, el cual también presenta vertebras anficélicas, muestra la combinación de características escapulares siguiente: 1) forma recta del margen anterodorsal, 2) proceso posterior reducido, 3) eje escapular presente proximal y distalmente, y 4) ángulo menor a $60^{\circ}$ entre el eje escapular y la faceta glenoidea. Estos caracteres figuran en la matriz de datos de Moon (2019), donde la combinación se presenta en diversos géneros de la familia Ophthalmosauridae, aunque también en algunos taxones del clado Thunnosauria.

$\mathrm{El}$ acromion, al no ser visible, dificulta la identificación del ejemplar, pues de acuerdo con Moon (2019), la prominencia del proceso acromial, en combinación con las características mencionadas anteriormente, se encuentran únicamente en los géneros de la familia Ophthalmosauridae.

\section{Conclusiones}

El ejemplar FCBUANL_2709 pertenece a un ictiosaurio, en el cual, la asignación taxonómica más detallada se ve comprometida debido al poco material que presenta el ejemplar, dejando su asignación al clado Thunnosauria. Asimismo, debido a sus medidas, las vértebras de FCBUANL_2709 corresponden a un ejemplar de grandes dimensiones.

Por su parte, el FCBUANL_2708 es un ictiosaurio de menor tamaño respecto al ejemplar FCBUANL_2709, que, gracias a la presencia de elementos apendiculares, puede ser asociado a la familia Ophthalmosauridae.

Los registros de reptiles marinos del noreste de México son importantes porque documentan su presencia en el país. Así, el presente reporte no 
debe de ser leído como una conclusión del estudio de los reptiles marinos del noreste de México. Al contrario, debe de tomarse como un indicador del vasto potencial de dicha región que debe de ser todavía apreciado. Sobran kilómetros cuadrados de yacimientos por ser explorados y varios especímenes por ser encontrados.

\section{Agradecimientos}

Agradecemos al Dr. David Lazcano Villarreal y Dr. José Rubén Guzmán Gutiérrez por su revisión y valiosos comentarios a este trabajo. Al M.C. Hidalgo Rodríguez Vela, por la realización de la cartografía. A los Sres. José Francisco Morales Silva y Lauro Zúñiga Sánchez (Comisariado Ejidal y Tesorero, respectivamente, del Ejido Pablillo, quienes apoyaron con los permisos para la extracción de los ejemplares).

\section{Referencias}

Adatte, T., Stinnesbeck, W., Remane, J., 1994, The Jurassic-Cretaceous boundary in Northeastern Mexico. Confrontation and correlations by microfacies, clay minerals mineralogy, calpionellids and ammonites: Geobios, 27, 37-56. https://doi. org/10.1016/s0016-6995 (94)80124-x

Barrientos-Lara, J. I., Alvarado-Ortega, J., 2020, Acuetzpalin carranzai gen et sp. nov. A new ophthalmosauridae (Ichthyosauria) from the Upper Jurassic of Durango, North Mexico: Journal of South American Earth Sciences, 98, 102456. https://doi.org/10.1016/j. jsames.2019.102456

Buchy, M. G., 2007, Mesozoic marine reptiles from north-east Mexico: description, systematics, assemblages and palaeobiogeography: Alemania, University of Karlsruhe, tesis doctoral, $87 \mathrm{p}$.

Buchy, M. G., 2010, First record of Ophthalmosaurus (Reptilia: Ichthyosauria) from the Tithonian (Upper Jurassic) of Mexico: Journal of Paleontology, 84(1), 149155. https://doi.org/10.1666/08-122.1

Buchy, M. C., Covarrubias Cervantes, A., 2011, Large ichthyosaurian remains from the La Casita type locality (Tithonian, Upper Jurassic), Coahuila, Mexico: Paludicola, 8(2), 100-105.

Buchy, M. C., López-Oliva,J. G., 2009, Occurrence of a second ichthyosaur genus (Reptilia: Ichthyosauria) in the Late Jurassic Gulf of Mexico: Boletín de la Sociedad Geológica Mexicana, 61(2), 233-238. https://doi. org/10.18268/bsgm2009v61n2al 1

Buchy, M. C., Frey, E., Stinnesbeck, W., LópezOliva, J. G., 2006, An annotated catalogue of the Upper Jurassic (Kimmeridgian and Tithonian) marine reptiles in the collections of the Universidad Autónoma de Nuevo León, Facultad de Ciencias de la Tierra, Linares, Mexico: Oryctos, 6, 1-18.

Caldwell, M. W., 1997, Modified perichondral ossification and the evolution of paddle-like limbs in ichthyosaurs and plesiosaurs: Journal of Vertebrate Paleontology, 17(3), 534-547. https://doi.org/10.1080/02724634.1997.1 0011000

Frey, E., Stinnesbeck, W., 2014, Mexican Ichthyosaurs, en Rivera-Sylva, H. E.; Carpenter, K. \& Frey, E. (eds.), Dinosaurs and Other Reptiles from the Mesozoic of Mexico: Bloomington, Indiana University Press, 30-43.

Imlay, R. W., 1939, Upper Jurassic ammonites from Mexico: Bulletin of the Geological Society of America, 50(1), 1-78. https://doi. org/10.1130/GSAB-50-1

Maisch, M. W., Matzke, A. T., 2000, The Ichthyosauria: Stuttgarter Beiträge zur Naturkunde,Serie B (Geologie und Paläontologie), 298, 1-159.

McGowan, C., Motani, R., 2003, Ichthyopterygia, en Hans-Dieter S. (ed.) Handbook of Paleoherpetology: Munich, Verlag Dr. Friedrich Pfeil, 175 p. 
Moon, B. C., 2019, A new phylogeny of ichthyosaurs (Reptilia: Diapsida): Journal of Systematic Palaeontology, 17(2), 129-155. https://doi.org/10.1080/14772019.2017.1394922

Motani, R., 1999, Phylogeny of the Ichthyopterygia: Journal of Vertebrate Paleontology, 19(3), 473-496. https://doi.org / 10.1080/02724634.1999.10011160

Rogers, C. L., De Ciserna, Z., Tavera, A. E., Ulloa, S., 1957, Geología general y depósitos de fosfatos del distrito de Concepción del Oro, estado de Zacatecas: Boletín del Instituto Nacional para la Investigación de Recursos Minerales, 38, 1-129. https://doi. org/10.3133/b1037a

Zell, P., Crame, J. A., Stinnesbeck, W., Beckmann, S., 2015, The bivalve Anopaea (Inoceramidae) from the Upper Jurassiclowermost Cretaceous of Mexico: Journal of South American Earth Sciences, 60, 92-103. http://dx.doi.org/10.1016/j. jsames.2015.03.004

Zell, P., Stinnesbeck, W., 2016, Paleobiology of the latest Tithonian (Late Jurassic) ammonite Salinites grossicostatum inferred from internal and external shell parameters: PLOS ONE, 11(1), e0145865. https://doi. org/10.1371/journal.pone.0145865 